Revista Arbitrada Interdisciplinaria KOINONIA

Año V. Vol V. Nº. Enero - Junio 2020

Hecho el depósito de Ley: FA2016000010 ISSN: 2542-3088

FUNDACIÓN KOINONIA (F.K). Santa Ana de Coro. Venezuela.

Adán Guillermo Ramírez-García

http://dx.doi.org/10.35381/r.k.v5i9.557

\title{
Las enseñanzas de Don Jesús: Una forma yaqui de agroecología
}

\section{The teachings of Don Jesus: A Yaqui form of agroecology}

\author{
Adán Guillermo Ramírez-García \\ aguillermoramirezgarcia@gmail.com \\ Universidad Autónoma Chapingo, Chapingo \\ México \\ https://orcid.org/0000-0002-1711-5942
}

Recibido: 15 de julio de 2019

Revisado: 05 de agosto de 2019

Aprobado: 25 de agosto de 2019

Publicado: 20 de enero de 2020

\section{RESUMEN}

El objetivo del presente trabajo fue realizar una aproximación para comparar el nivel de sustentabilidad entre el sistema monocultivo trigo y un sistema diversificado presentes en el Valle del Yaqui, lugar donde nació la llamada Revolución Verde y se encuentra localizado en el Sur de Sonora, México. Los ejes del análisis son la innovación, el consumo del agua y la rentabilidad. La metodología aplicada para la realización del presente estudio es una modificación al Marco para la Evaluación de Sistemas de Manejo de Recursos Naturales Incorporando Indicadores de Sustentabilidad (MESMIS). Los resultados indican que, de los 13 indicadores de sustentabilidad evaluados, el sistema diversificado presenta más indicios de sustentabilidad.

Descriptores: Ecosistema acuático; agua; recursos alimentarios; seguridad alimentaria. (Palabras tomadas del Tesauro UNESCO).

\begin{abstract}
The objective of this work was to make an approximation to compare the level of sustainability between the wheat monoculture system and a diversified system present in the Yaqui Valley, where the so-called Green Revolution was born and which is located in Southern Sonora, Mexico. The axes of the analysis are innovation, water consumption and profitability. The methodology applied to carry out this study is a modification to the Framework for the Evaluation of Natural Resource Management Systems Incorporating Sustainability Indicators (MESMIS). The results
\end{abstract}




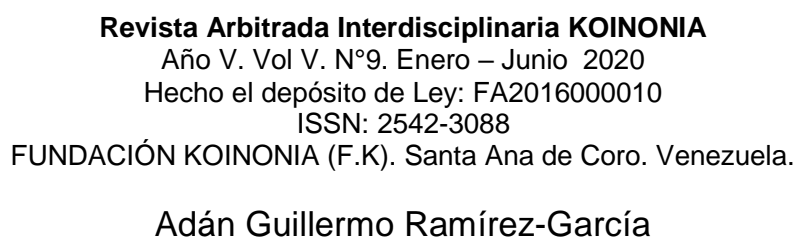

indicate that, of the 13 sustainability indicators evaluated, the diversified system presents more signs of sustainability.

Descriptors: Aquatic ecosystems; water; food resources; food security. (Words taken from the UNESCO Thesaurus).

\section{INTRODUCCIÓN}

Sin duda, el hombre ha ejercido presión en la biósfera con consecuencias irreversibles con la finalidad de satisfacer sus necesidades; basta señalar que las necesidades de 7.6 mil millones de personas en el planeta exigen incrementar la tasa de utilización de los recursos aprovechados a través de la agricultura, ganadería, industria, pesca y comercio (Altieri, 1999).

En lo que se refiere a la actividad agrícola y sus impactos, el Valle del Yaqui, ubicado en el Sur del estado de Sonora, México, representa un buen ejemplo. El desarrollo de la investigación agrícola en el Valle del Yaqui comenzó en 1910 por parte de la compañía Richardson (Okada, 2000); en 1930 se inicia la investigación con trigo, arroz y ajonjolí; en 1945 el Doctor Norman E. Borlaug realizó los primeros ensayos de adaptación de líneas mejoradas de trigo (Picado, 2012) y con ello se inicia la llamada Revolución Verde (RV). La RV fue un movimiento con impacto inmediato internacional y trascendió las fronteras estatales y nacionales para llegar, con las variedades mejoradas del trigo, a muchos países en desarrollo. Las variedades mejoradas en el Valle del Yaqui, fueron enviadas a otras partes del planeta, logrando incrementar la producción de granos en India, Pakistán y Bangladesh. En el ámbito mundial, 60 millones de hectáreas se siembran con variedades de origen mexicano provenientes de este valle, (INIFAP, 1999).

El Valle del Yaqui fue considerado como uno de los más fértiles y productivos de todo el país, por lo que se denominó, "el granero de México", destacando el trigo (Evans, 2006). Sus tierras fueron utilizadas para generar e impulsar la tecnología agrícola moderna lo que vendría a llamarse "Revolución Verde", cuya tecnología innovadora permitió el incremento en los rendimientos de los cultivos, desde mediados del siglo pasado hasta la fecha. 


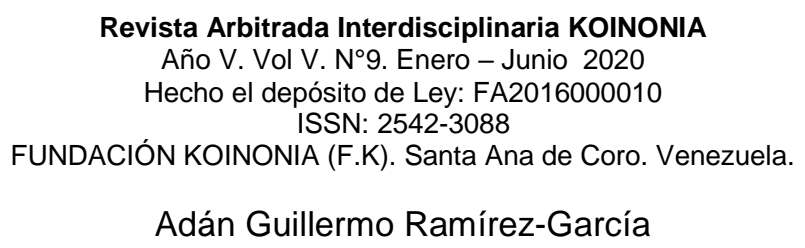

Además del mejoramiento genético en las variedades de trigo, la toponimia del valle como granero de México se logró gracias al uso de fertilizantes de fuentes inorgánicas, plaguicidas, maquinaria agrícola y, principalmente, semillas mejoradas. A esto puede agregarse los ciclos más o menos regulares de lluvias, pero, sobre todo, por la infraestructura hidráulica que se empezó a crear desde finales del siglo XIX y principios del XX (Padilla, 2014), reiniciándose en la década de los treinta, continuándose a finales de los cuarenta y principios de los cincuenta con la construcción de la presa "Álvaro Obregón" lo que permitió un mayor almacenamiento y un mejor aprovechamiento y distribución del agua, (INIFAP, 1999).

La agricultura del Valle del Yaqui es un representante fiel de lo que es la agricultura bajo el modelo de la RV. Por lo que, pensar en el futuro de la agricultura en el Valle del Yaqui es una situación relevante, sobre todo cuando se consideran los factores económicos, sociales y ambientales que implican el desarrollo de la producción primaria; no se puede pensar en el desarrollo de una agricultura con carácter sustentable si no se contemplan dichos componentes, los cuales interactúan entre sí y no deben observarse por separado, (Sánchez, 2005).

Actualmente, de acuerdo con Borbón, et al., (2011), en el caso particular del Valle del Yaqui los problemas que dificultan el desenvolvimiento de la actividad agrícola son: el poco financiamiento, la falta de apoyos gubernamentales y los asociados a la comercialización. Al no contar con recursos de financiamiento se fomenta el agiotismo, el rentismo de la tierra que degenera en un abandono eventual de la actividad y el acaparamiento de los medios de producción. Con el fracaso de la unidad de producción se conduce a una perdida en el nivel de empleo y se ven obligados muchos de los pequeños productores a la migración hacia otros lugares. En un contexto más amplio, al disminuir la producción se estimula la mayor importación de granos básicos. Por su parte, Berber et al., (2014), menciona que otro problema a considerar es la diferencia de los precios, que en términos reales han disminuido y que los insumos utilizados para su producción han aumentado considerablemente.

En este sentido desde hace un tiempo se ha planteado la necesidad de transitar hacia una agricultura sustentable; sin embargo, de acuerdo con Sevilla (2001), 


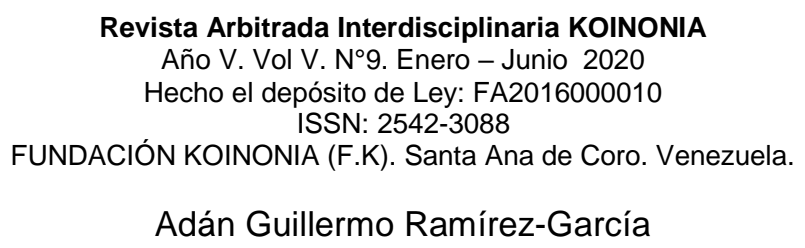

resulta imposible dar una definición universal sobre agricultura sustentable debido a que es un concepto referido a un proceso dinámico, cambiante en el tiempo, con su escala espacial, con las preocupaciones de cada época, con el desarrollo de la ciencia y con el nivel de conocimiento actual.

De acuerdo con Castillo et al. (2012), dentro de la agricultura se expresa una de las más dinámicas y complejas interrelaciones de los aspectos biofísicos y socioeconómicos, estas condiciones han creado la necesidad de buscar perspectivas que abarquen de forma integral todos los componentes.

Por ello, es necesario incorporar métodos prácticos que permitan la evaluación de la sustentabilidad que no solo expliquen el funcionamiento de un sistema y los efectos de adopción o no de tecnología, sino que muestren y expliquen cómo interactúan las dimensiones sociales, económicas y ambientales para permitir la operación y desarrollo del sistema de producción (Brunett, 2004).

Un término asociado a la agricultura sustentable tiene que ver con el desarrollo sustentable; y al igual para la agricultura sustentable, no existe una definición consensuada del término que nos ocupa. Bermejo (2014), al respecto define el desarrollo sustentable como el manejo y conservación de los recursos naturales orientados a provocar cambios tecnológicos e institucionales que aseguren la satisfacción de las necesidades humanas para las presentes y futuras generaciones de una forma continuada. Tal desarrollo busca conservar el suelo, el agua, los recursos genéticos animales y vegetales; no degradando al medio ambiente.

En la perspectiva anterior, Gliessman (2002), argumenta que una agricultura sustentable es aquella que, como mínimo, logra el menor efecto negativo en el ambiente, preserva y reconstruye la fertilidad del suelo, usa el agua de manera eficiente, hace uso de los recursos dentro del agroecosistema reemplazando los insumos externos, valorando y conservando la biodiversidad biológica y garantizando la equidad en el acceso a las prácticas agrícolas apropiadas al conocimiento y a la tecnología. Igualmente afirma que una agricultura sustentable debe procurar el control local de los recursos agrícolas.

Entre esta y muchas otras definiciones que existen de agricultura sustentable, se puede decir que existen ciertos objetivos comunes en la mayoría de ellas: buscan 
producción estable procurando usar eficientemente los recursos, seguridad y autosuficiencia alimentaria, usar prácticas agroecológicas o tradicionales de manejo, preservar la cultura local y de la pequeña propiedad, asistencia de los más pobres a través de un proceso de autogestión, alto nivel de participación de la comunidad en decidir la dirección de su propio desarrollo agrícola y la conservación y regeneración de los recursos naturales (Altieri y Nicholls, 2000). Para los fines concretos de este trabajo se considerará a la agricultura sustentable como aquella que intenta proporcionar rendimientos sostenidos a largo plazo, utilizando la diversificación productiva y usando tecnologías de manejo que integren todos los componentes del predio para mejorar la eficiencia biológica del sistema, manteniendo la capacidad productiva del agroecosistema, conservando la biodiversidad, generando condiciones favorables para que el sistema se autorregule $y$, por otro lado, promueve la participación social procurando el mejoramiento socioeconómico del productor.

Este trabajo tiene la finalidad de llevar a cabo la propuesta metodológica del Marco para la Evaluación de Sistemas de Manejo de recursos naturales incorporando Indicadores de Sostenibilidad (MESMIS) (Masera, Astier y López, 2000); el trabajo realizará un estudio comparativo entre la producción de trigo bajo el esquema de la $\mathrm{RV}$ y un sistema diversificado basado en principios agroecológicos.

Se elige el trigo porque Sonora es un estado que aporta el 50 por ciento de la producción nacional y representa el 38 por ciento de la superficie cultivada en México. La gran producción triguera sonorense se debe a una conjunción de factores: condiciones climáticas favorables, abundancia de tierras de buena calidad, con riego y prácticas mecanizadas, apoyo gubernamental, subsidios, apoyos a la comercialización, mejoramiento continuo de la infraestructura y otras políticas de apoyo. Además, y lo más importante, los productores emprendedores que han aprovechado adecuadamente estos factores (Márquez, et al., 2014).

México es deficitario en trigo, pero la mayoría del trigo sonorense no corresponde a las requeridas por el mercado, lo que implica la necesidad de exportarlo o de buscar otros usos, como el forraje. 
Existe la urgente necesidad de identificar opciones de cultivo que puedan tener una buena adaptación a las condiciones agroclimáticas del Sur de Sonora, para posteriormente, desarrollar el paquete tecnológico que pueda proporcionar rendimientos competitivos en costo con otras regiones del país o del mundo; sobre todo, identificar las opciones de comercialización rentable para estos productos. Respecto al sistema diversificado con el que se contrastará la producción de trigo se puede considerar como un sistema de innovación en agricultura.

Los sistemas de innovación en agricultura son redes de instituciones, empresas, organizaciones e individuos que solicitan y ofrecen conocimientos y tecnologías, y se orientan a la utilización de nuevos productos, procesos y formas de organización y a las reglas y los mecanismos por medio de los cuales ellos interactúan (Sonino y Ruane, 2011). La combinación de cultivos es una manera de innovar en la agricultura ya que es mucho más fácil poder optimizar el espacio para poder tener una amplia variedad de productos y con mayor seguridad de buenos resultados.

El objetivo del presente trabajo es realizar una aproximación para comparar el nivel de sustentabilidad entre el sistema monocultivo trigo en relación con un sistema diversificado. Los ejes del análisis son la innovación, el consumo del agua y la rentabilidad.

\section{MATERIALES Y MÉTODOS}

El Valle del Yaqui es una de las principales regiones agrícolas de México y se encuentra en el sur del estado de Sonora, México, entre la Sierra Madre Occidental y el Mar de Cortés; el valle se ubica, parcialmente, en los territorios de los municipios de Cajeme, Bácum, San Ignacio Río Muerto, Benito Juárez, Etchojoa y Navojoa. Al norte, el valle colinda con Ciudad Obregón y al sur limita con el Valle del río Mayo; el valle se ubica entre los paralelos $27^{\circ} 10^{\prime}$ y $27^{\circ} 50^{\prime}$ latitud norte, y los meridianos $109^{\circ} 55^{\prime}$ y $110^{\circ} 36^{\prime}$. 
Adán Guillermo Ramírez-García

Localización del área de estudio.

Figura 1

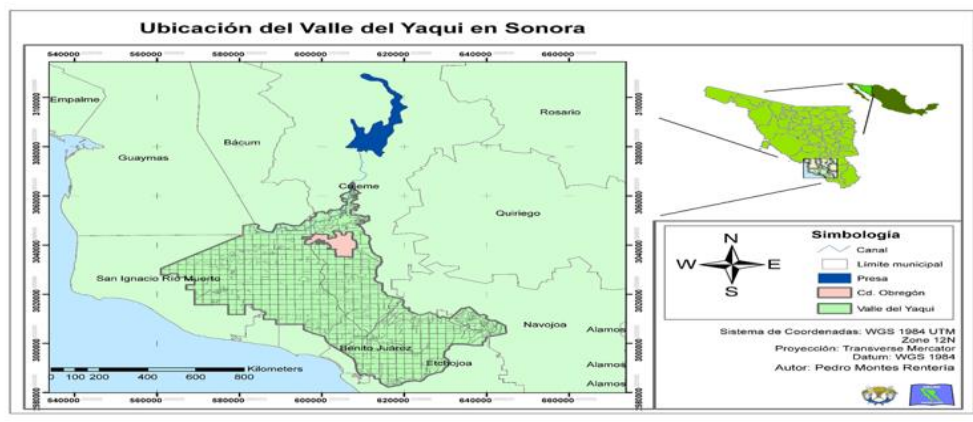

Fuente: Elaboración propia

La metodología aplicada en la realización del presente estudio es la propuesta MESMIS por Masera, Astier y López (2000). Se parte de considerar que para lograr la sustentabilidad de un agroecosistema por lo menos se debe contar con los siguientes atributos o propiedades sistémicas fundamentales por (Conway, 1989):

a) Productividad. Es la habilidad del agroecosistema para proveer el nivel requerido de bienes y servicios.

b) Confiabilidad. Se refiere a la capacidad del sistema de mantenerse en niveles cercanos al equilibrio ante perturbaciones normales del ambiente.

c) Estabilidad. Es la propiedad del sistema de tener un estado de equilibrio dinámico estable.

d) Resiliencia. Es la capacidad de retomar al estado de equilibrio o mantener el potencial productivo después de que el sistema haya sufrido perturbaciones graves.

e) Adaptabilidad. Es la capacidad del sistema de encontrar nuevos niveles de equilibrio ante cambios de largo plazo en el ambiente.

f) Equidad. Es la habilidad del sistema para distribuir la productividad (beneficios o costos) de una manera justa, y

g) Autogestión: Se refiere a que el sistema posee un nivel aceptable para responder y controlar los cambios inducidos desde el exterior. 
Revista Arbitrada Interdisciplinaria KOINONIA

Año V. Vol V. No9. Enero - Junio 2020

Hecho el depósito de Ley: FA2016000010 ISSN: 2542-3088

FUNDACIÓN KOINONIA (F.K). Santa Ana de Coro. Venezuela.

Adán Guillermo Ramírez-García

\section{RESULTADOS Y DISCUSIÓN}

\section{Determinación del objeto de estudio}

El objeto de estudio de este trabajo es comparar el cultivo de trigo bajo el modelo intensivo Revolución Verde con un sistema de producción diversificado en el Valle del Yaqui.

\section{Cuadro 1}

Comparación de las determinantes de los predios en estudio

\begin{tabular}{|c|c|c|}
\hline DETERMINANTES & CULTIVO DIVERSIFICADO & MONOCULTIVO \\
\hline Superficie & 4ha & 4ha \\
\hline \multirow[t]{3}{*}{ Propietario } & Don Jesús Higuera 65 años & 58 años \\
\hline & Cultivo diversificado & Monocultivo Trigo-Maíz \\
\hline & $\begin{array}{l}\text { Subsistema agrícola: nopal } \\
\text { (Opuntia ficus-indica L. Miller) } \\
\text { variedades Copena y Misión. } \\
\text { Subsistemas frutales: higo } \\
\text { (Ficus garica) y naranja (Citrus } \\
\text { arantium var. Sinensis). } \\
\text { Subsistema ornamental: } \\
\text { variedad de rosas (Rosa sp) } \\
\text { Sistema avícola: gallinas (Gallus } \\
\text { gallus domesticus) y guajolotes }\end{array}$ & $\begin{array}{l}\text { Las variedades de trigo más utilizadas } \\
\text { en el Valle del Yaqui son: CIRNO } \\
\text { C2008, Villa Juárez Baviácora y } \\
\text { Aconchi. } \\
\text { Prácticas agronómicas recomendadas } \\
\text { en la región: preparación del terreno, } \\
\text { siembra, fertilización, riegos, control de } \\
\text { plagas, malezas y enfermedades, } \\
\text { cosecha y comercialización (FIRA, } \\
\text { 2018). }\end{array}$ \\
\hline \multirow{8}{*}{$\begin{array}{l}\text { Tecnológicas y de } \\
\text { manejo }\end{array}$} & (Meleagris gallopavo). & Se establece un cultivo al año (ciclo \\
\hline & Prácticas agronómicas son: & otoño-invierno). \\
\hline & $\begin{array}{l}\text { siembra, fertilización, poda, } \\
\text { riego, acodos, manejo de } \\
\text { plagas, cosecha. }\end{array}$ & $\begin{array}{l}\text { Tecnología empleada: mecanizada. } \\
\text { La fertilización del trigo es de origen } \\
\text { químico; los agroquímicos se utilizan }\end{array}$ \\
\hline & $\begin{array}{l}\text { Tecnología empleada: mixta } \\
\text { (manual y mecanizada) para el } \\
\text { establecimiento de los cultivos y }\end{array}$ & $\begin{array}{l}\text { intensivamente. } \\
\text { Mano de obra muy poca durante todo } \\
\text { el ciclo de cultivo. }\end{array}$ \\
\hline & manual para realizar las & Manejo de suelos. \\
\hline & prácticas agronómicas en los & No hay prácticas de conservación. \\
\hline & cultivos. Para el higo, el manejo & Manejo de plagas y enfermedades. \\
\hline & $\begin{array}{l}\text { agronómico es manual; el } \\
\text { tractor cada dos meses realiza }\end{array}$ & Químico. \\
\hline
\end{tabular}


Año V. Vol V. No9. Enero - Junio 2020

Hecho el depósito de Ley: FA2016000010 ISSN: 2542-3088

FUNDACIÓN KOINONIA (F.K). Santa Ana de Coro. Venezuela.

Adán Guillermo Ramírez-García

labores de descompactación del suelo mediante un paso de rastra. La fertilidad de los sueles se mejora por la adición de lixiviado de lombriz y por la anexión de potasio líquido. Mano de obra empleada: temporal y permanente (se tienen 3 empleos permanentes).

Manejo de suelos

Prácticas de conservación son abono orgánico, lixiviado de lombriz, cercos vivos.

Manejo de plagas $y$ enfermedades.

MIP (manual, químico y orgánico).

Modo de apropiación del terreno: pequeña propiedad sin reconocimiento jurídico.

\section{Socioeconómicas y culturales}

Características del objetivo de la producción:

Autoconsumo e ingresos por excedentes. La escala de producción es de 4 ha.
Modo de apropiación de las parcelas: pequeña propiedad y organizado en diversas figuras jurídicas.

Características del objetivo de la producción:

Se buscan ingresos económicos maximizados.

Fuente: Elaboración propia.

\section{Identificación de los puntos críticos}

Los puntos críticos en el cultivo del trigo en el Valle del Yaqui son: alta demanda de fertilizantes, uso intensivo de maquinaria agrícola, riego por gravedad, uso indiscriminado de herbicidas, pesticidas, y fungicidas, entre otros. Sin embargo, el uso indiscriminado de los agroquímicos, durante estas siete décadas, ha llevado al Valle del Yaqui a su deterioro ambiental, caracterizado por la contaminación del agua, aire y suelo, provocando efectos nocivos en la fauna silvestre y en la salud humana, además de aumentar la vulnerabilidad de los cultivos a los agentes 
Año V. Vol V. No9. Enero - Junio 2020

Hecho el depósito de Ley: FA2016000010 ISSN: 2542-3088

FUNDACIÓN KOINONIA (F.K). Santa Ana de Coro. Venezuela.

Adán Guillermo Ramírez-García

patógenos y la desaparición de especies que formaban parte de la biodiversidad agrícola (Fabio et al., 2002); además, en el suelo se ha observado ensalitramiento, compactación, empobrecimiento, erosión y contaminación del suelo, desplazamiento de especies animales y vegetales nativas, contaminación y abatimiento de los mantos freáticos y desocupación de mano de obra por la utilización de maquinaria agrícola (Almanza, 2008).

\section{Selección y medición de indicadores estratégicos}

De acuerdo con los puntos críticos identificados, los atributos, criterios de diagnóstico indicadores, definición de cada indicador y la fórmula para su cálculo que se tomó en cuenta para evaluar cada uno de los sistemas son señalados se señala en el cuadro 2

\section{Cuadro 2}

Indicadores estratégicos

\begin{tabular}{|c|c|c|c|c|}
\hline Atributo & $\begin{array}{l}\text { Criterio de } \\
\text { diagnóstico }\end{array}$ & $\begin{array}{c}\text { Indicadores } \\
\text { Seleccionados }\end{array}$ & Definición & Formula \\
\hline $\begin{array}{l}\text { Productivid } \\
\text { ad }\end{array}$ & Eficiencia & $\begin{array}{l}\text { Relación } \\
\text { costo/beneficio }\end{array}$ & $\begin{array}{l}\text { Relación } \\
\text { entre costos } \\
\text { y beneficios } \\
\text { asociados a } \\
\text { un proyecto } \\
\text { de inversión. }\end{array}$ & $\mathrm{B} / \mathrm{C}=\mathrm{INA} / \mathrm{ENA}$ \\
\hline & & & $\begin{array}{l}\text { Biodiversida } \\
\text { d más } \\
\text { aparente y } \\
\text { que se capta }\end{array}$ & $\begin{array}{l}\text { Número de especies presentes y } \\
\text { número de cultivos que generan } \\
\text { ingreso económico en el predio. }\end{array}$ \\
\hline $\begin{array}{l}\text { Estabilidad, } \\
\text { Resiliencia, } \\
\text { Confiabilida } \\
\text { d }\end{array}$ & $\begin{array}{l}\text { Diversidad } \\
\text { específica y } \\
\text { económica }\end{array}$ & $\begin{array}{l}\text { Especies } \\
\text { presentes y } \\
\text { económicament } \\
\text { e útiles }\end{array}$ & $\begin{array}{l}\text { a simple } \\
\text { vista. } \\
\text { La } \\
\text { diversidad } \\
\text { económica } \\
\text { se refiere a } \\
\text { la cantidad }\end{array}$ & \\
\hline
\end{tabular}


Revista Arbitrada Interdisciplinaria KOINONIA

Año V. Vol V. Nº . Enero - Junio 2020

Hecho el depósito de Ley: FA2016000010

ISSN: 2542-3088

FUNDACIÓN KOINONIA (F.K). Santa Ana de Coro. Venezuela.

Adán Guillermo Ramírez-García

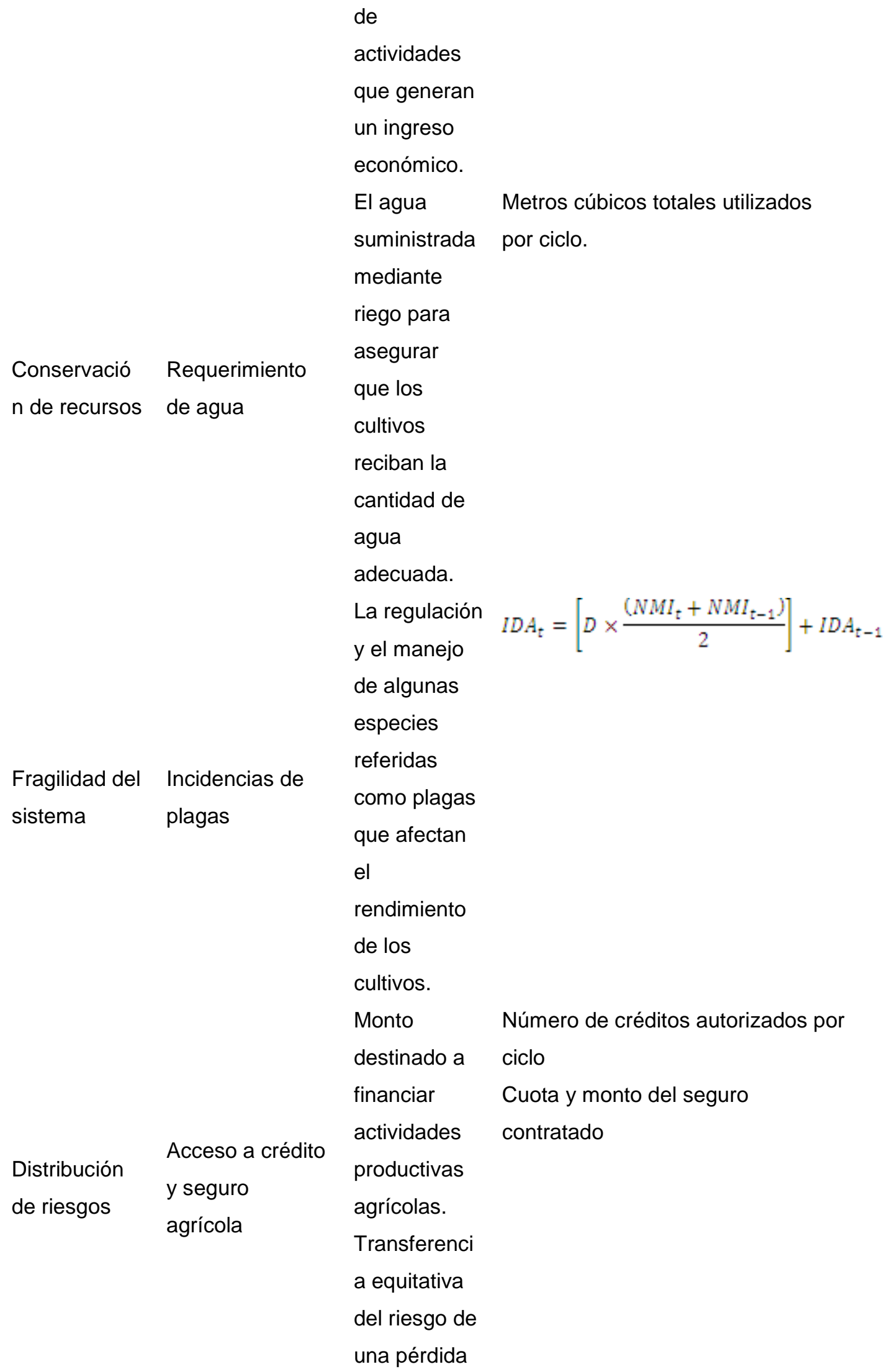


Revista Arbitrada Interdisciplinaria KOINONIA

Año V. Vol V. N9. Enero - Junio 2020

Hecho el depósito de Ley: FA2016000010

ISSN: 2542-3088

FUNDACIÓN KOINONIA (F.K). Santa Ana de Coro. Venezuela.

Adán Guillermo Ramírez-García

de una

entidad a

otra a

cambio de

una prima.

Adopción de Número de prácticas innovadoras

nuevas

implementadas

tecnología,

prácticas,

conocimient

Adaptabilid

Capacidad
de cambio e
innovación

Generación de

os y

ad

conocimientos y

nuevas prácticas

Distribución

de costos y

beneficios

Equidad

\section{Beneficiarios \\ directos}

Demanda de
trabajo

Integración de

Los

beneficiarios en

las fases del

cultivo

los

\section{esquemas}

mentales, de

nuevas

instituciones,

formas de

organización

social y

mercados

Personas que reciben

beneficios

del sistema

de cultivo

Cantidad de Número de personas que laboran, personas permanente y temporalmente, en el que tienen sistema de producción.

empleo

beneficiarios

Número de personas que participan

participan en

los procesos

de trabajo

en el cultivo. 
Revista Arbitrada Interdisciplinaria KOINONIA

Año V. Vol V. Nº. Enero - Junio 2020

Hecho el depósito de Ley: FA2016000010

ISSN: 2542-3088

FUNDACIÓN KOINONIA (F.K). Santa Ana de Coro. Venezuela.

Adán Guillermo Ramírez-García

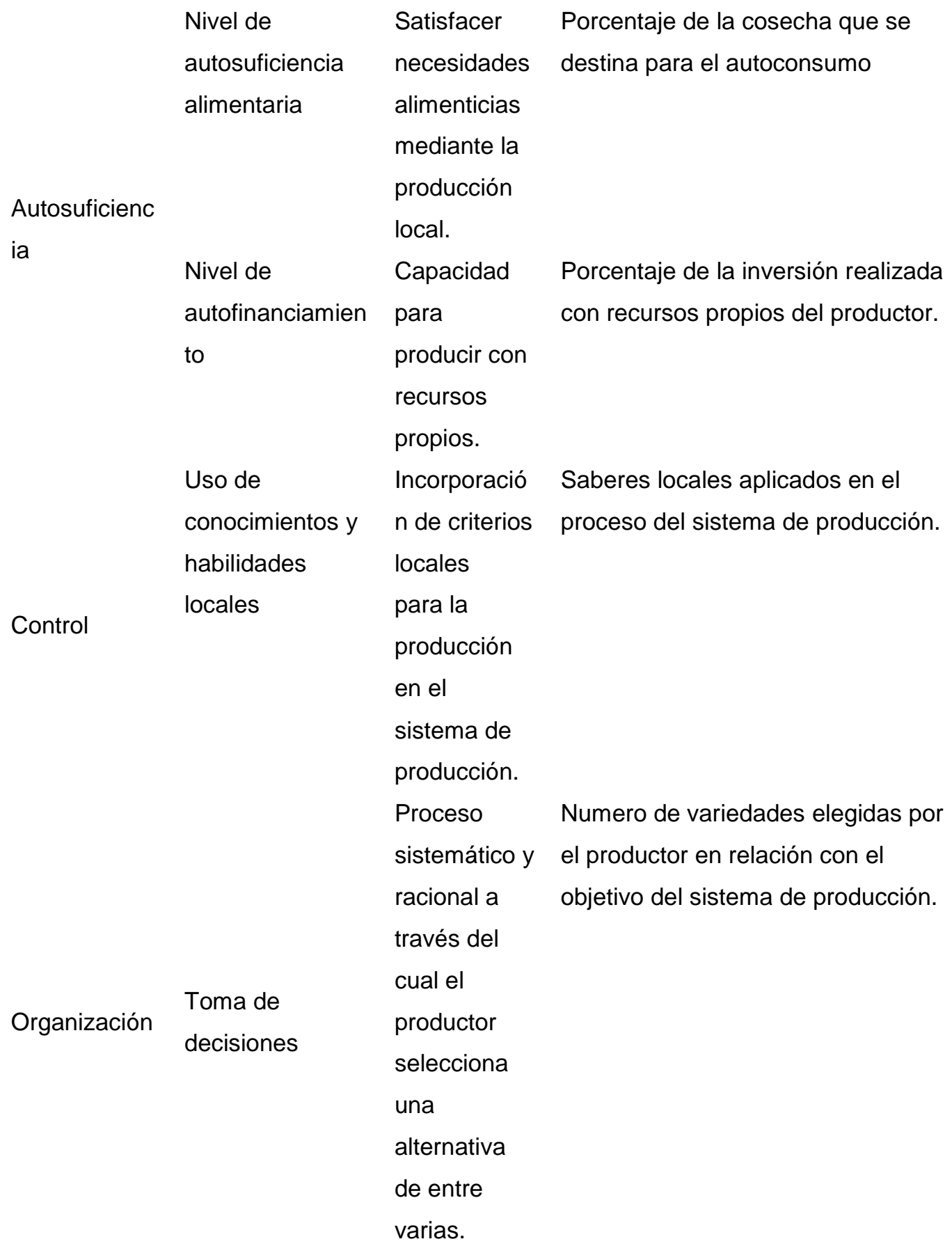

Donde INA: ingresos netos actualizados a una tasa de referencia; ENA: egresos netos actualizados a una tasa de referencia; $I_{D A}=$ número de individuos-día acumulados en el

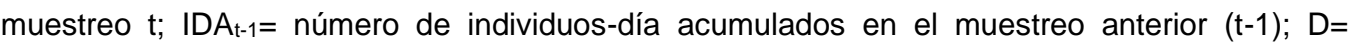
número de días entre el muestreo (t-1) y $\mathrm{t} ; \mathrm{NMl}_{\mathrm{t}}=$ número medio de individuos en el muestreo $\mathrm{t}$; $\mathrm{NMI}_{\mathrm{t}-1}=$ número medio de individuos en el muestreo (t-1) (Moreno Vázquez, 1994a,1994b; Cabello y Benítez, 1994).

\section{Fuente: Elaboración propia.}


Adán Guillermo Ramírez-García

\section{Medición y monitoreo de los indicadores}

Los datos y la información recabada fueron recopilados de diferentes fuentes. En el caso del sistema de referencia trigo se realizaron entrevistas y una revisión bibliográfica de las páginas web de FIRA (2018) y DRRY (2018); mientras, para el sistema diversificado, la obtención de los datos fue lograda a través de una entrevista con Don Jesús y de recorridos de campo en el predio.

\section{Integración de resultados de la comparación de la sustentabilidad}

A partir de los resultados descritos anteriormente, desde el numeral 1 hasta el 13, la evaluación de ambos sistemas (monocultivo versus sistema diversificado) permiten la comparación de dos sistemas de producción analizado cualitativamente convertido a un análisis cuantitativo. En el cuadro 3, se presentan las estimaciones cuantitativas de ambos sistemas comparados con un modelo deseable, modelo diseñado ex profesamente para este trabajo. A partir de la evaluación cualitativa y con la finalidad de graficar los resultados, en este trabajo se propone utilizar la escala del 1 al 5 para comparar los sistemas de producción analizados.

\section{Cuadro 3.}

Análisis de la sustentabilidad de los sistemas diversificado vs monocultivo.

Indicador
Sistema Monocultiv Deseabl diversificad

o

Relación costo/beneficio

Especies presentes y económicamente útiles

Volumen de agua utilizado

Incidencias de plagas y enfermedades

Acceso a créditos y seguros

Generación de conocimientos y prácticas

Números de beneficiarios directos

Generación de empleos

$\begin{array}{lll}5 & 2 & 5 \\ 5 & 2 & 5 \\ 3 & 3 & 1 \\ 2 & 5 & 1 \\ 2 & 4 & 1 \\ 4 & 2 & 5 \\ 4 & 2 & 5 \\ 5 & 3 & 5\end{array}$


Año V. Vol V. No9. Enero - Junio 2020

Hecho el depósito de Ley: FA2016000010

ISSN: 2542-3088

FUNDACIÓN KOINONIA (F.K). Santa Ana de Coro. Venezuela.

Adán Guillermo Ramírez-García

Participación de los beneficiarios en las

distintas fases del cultivo

Nivel de autosuficiencia alimentaria

$5 \quad 2 \quad 5$

Nivel de autofinanciamiento

$4 \quad 2 \quad 5$

Uso de conocimientos y habilidades locales

42

Toma de decisiones

Fuente: Elaboración propia.

A continuación se visualizan las áreas de oportunidad de ambos sistemas de producción en el valle del Yaqui.

El sistema diversificado se acerca más al modelo deseable de produccion sustentable para la región del Valle del Yaqui. Es importante señalar que se identifican áreas de mejora para ambos sistenas de producción. Sin embargo, desde el punto de vista de la sustentabilidad, el sistema diversificado ofrece mayores ventajas respecto al monocultivo. 


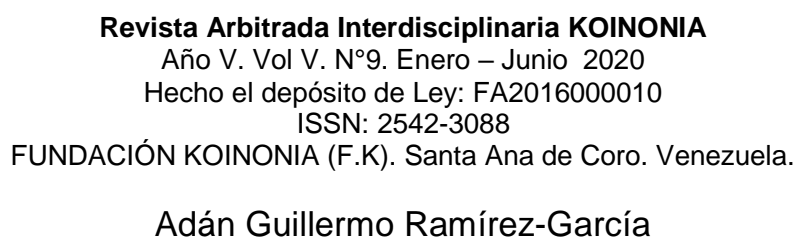

Figura 2

Evaluación de los puntos criticos

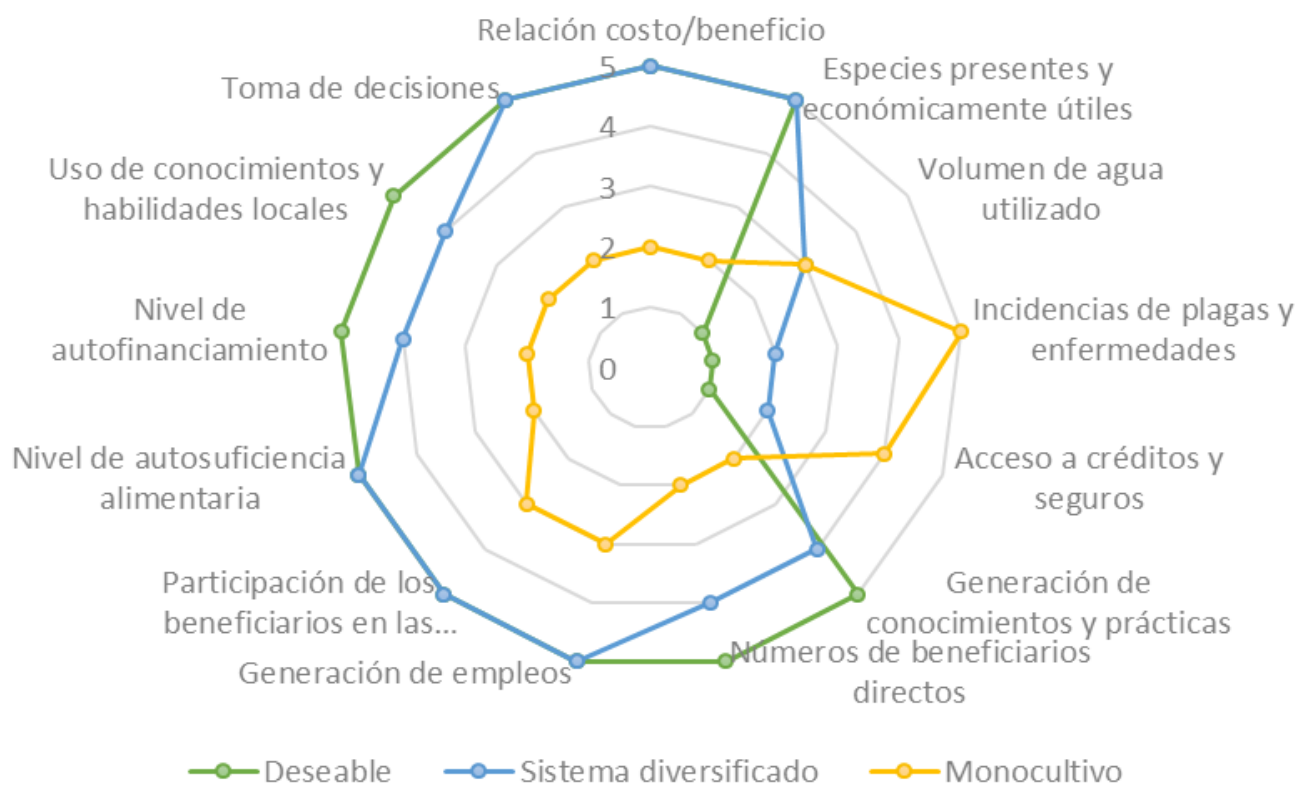

Fuente: Elaboración propia

\section{CONCLUSIONES}

Respecto al monocultivo donde se utiliza la tecnología tipo "Revolución Verde", que ocupa un alto insumo de agroquímicos y maquinaria que implica una importante inversión, además de que existe el peligro de la presencia de plagas o enfermedades que puedan dañar al cultivo presentando susceptibilidad al daño, además, no genera empleo a la población y el paquete tecnológico afecta la calidad del aire, suelo y agua de la región.

Don Jesús Higuera un agricultor de 65 años, quien siempre ha vivido en el Valle del Yaqui, ha creado una innovación en su predio en solo 4 hectáreas, rodeado en una región donde el sistema de monocultivo impera y modela la producción agrícola. La diversificación de cultivos, puesto en práctica por Don Jesús, se considera innovador, en comparación con el sistema convencional del trigo en el Valle del Yaqui ya que posee amplias ventajas tanto económicas como ambientales respecto al monocultivo. 
Relevante es mencionar que el productor, dueño de la idea del sistema diversificado, considera importante que otras personas compartan sus experiencias en la producción de cultivos alternativos porque precisamente ahí radica el enriquecimiento de la idea y apoya en la integración de nuevas ideas a su proyecto de trabajo. La enseñanza aprendida del sistema diversificado es que la apertura a nuevas ideas y aprendizajes permite obtener buenos resultados y con posibilidades de replicarse en la región como alternativa al desarrollo y la integración de la comunidad en un proyecto con múltiples beneficios.

A partir de la relación beneficio/costo, el sistema diversificado tiene mayor rentabilidad y autosostenibilidad. Complementarlo con actividades como cría de animales, procesamiento de materia prima cosechada (agregar valor agregado) y utilización de desechos orgánicos, traerá consigo un complemento perfecto para dejar de lado el uso de diversos insumos y desestimar esos costos, incrementando considerablemente el beneficio propio, la oferta de trabajo y ayudando a disminuir el impacto ambiental. Don Jesús desea, a futuro, ser un emprendedor empresarial, que comercialice productos terminados derivados de sus futuras cosechas, con un nombre de marca y normatividad cumplida para, inclusive, exportar. Este sistema diversificado es un claro ejemplo de innovación y emprendimiento, rompiendo estándares, disminuyendo actividades agrícolas y obteniendo una rentabilidad alta, deseable para cualquier agricultor, en este sentido, Salón\& Isea Argüelles (2019), destacan la importancia de contar con emprendimientos rurales con la finalidad de promover la soberanía agroalimentaria de los países.

\section{FINANCIAMIENTO}

No monetario.

\section{AGRADECIMIENTOS}

A la Universidad Autónoma Chapingo, a Don Jesús Higuera, proveedor de la información necesaria de su experiencia de campo y quien desinteresadamente participó brindando su experiencia y conocimientos para llevar a cabo este trabajo. 
Revista Arbitrada Interdisciplinaria KOINONIA

Año V. Vol V. Nº. Enero - Junio 2020

Hecho el depósito de Ley: FA2016000010 ISSN: 2542-3088

FUNDACIÓN KOINONIA (F.K). Santa Ana de Coro. Venezuela.

Adán Guillermo Ramírez-García

\section{REFERENCIAS CONSULTADAS}

Almanza, S. M. (2008). Las organizaciones del sector social del Valle del Yaqui. Retrocesos de política agraria. [The organizations of the social sector in Yaqui Valley. Setbacks of agricultural policy]. Frontera Norte, Vol. 20, Núm. 40, 33 p.

Altieri, M. (1999). Agroecología: Bases científicas para una agricultura Sustentable. [Scientific basis for sustainable agriculture]. Uruguay: Ed. Nordan-Comunidad.

Altieri, M. y Nicholls, C. (2000). Agroecología: Teoría y Práctica para una Agricultura Sostenible. [Agroecology: Theory and Practice for Sustainable Agriculture]. Serie Textos Básicos para la Formación Ambiental. ONU-PNUMA.

Berber, D. S., Almaguer Vargas, G., Schwentesius Rindermann, R., \& Ayala Garay, A. V. (2014). Trigo en Sonora y su contexto nacional e internacional. [Wheat in Sonora and its national and international context]. Obregón, Sonora, México.

Bermejo, G. R. (2014). Del desarrollo sostenible según Brundtland a la sostenibilidad como biomimesis. [From sustainable development according to Brundtland to sustainability as biomimesis]. Universidad del País Vasco. España. 60 p.

Borbón, C. (2011). Diagnóstico del Sector Agropecuario y Pesquero del Estado de Sonora. [Diagnosis of the Agricultural and Fishing Sector of the Sonora State]. Hermosillo, Sonora: Centro de Investigación en Alimentación y Desarrollo, A.C.

Brunett P., L. (2004). Contribución a la evaluación de la sustentabilidad: estudio de casos de dos agroecosistemas de producción de leche del Valle de Toluca. [Contribution to the evaluation of sustainability: case study of two agroecosystems for milk production in the Toluca Valley]. Tesis Doctoral Facultad de Medicina Veterinaria y Zootecnia, Universidad Nacional Autónoma de México. México.

Cabello, T., y Benítez E. (1994). Frankliniella occidentalis (Thysanoptera; Thripidae). En: Moreno, $\mathrm{R}(\mathrm{Ed}$.). [Frankliniella occidentalis (Thysanoptera; Thripidae). In: Moreno, $\mathrm{R}$ (Ed.). Plant health in protected horticulture]. Sanidad vegetal en la horticultura protegida. Consejería de Agricultura y Pesca. Junta de Andalucía. Sevilla: 243-259.

Castillo, D. et al. (2012). Evaluación de la sustentabilidad social, económica y productiva de dos agroecosistemas de producción de leche en pequeña escala en el municipio de Amecameca, México. [Evaluation of the social, economic and productive sustainability of two small-scale milk production agroecosystems in the municipality of Amecameca, Mexico]. Revista Científica UDO Agrícola. Vol. 12, №. 3, 2012, págs. 690-704

DRRY. (2018). Historia del Distrito de Riego del Rio Yaqui (DRRY). ). [History of the Yaqui River Irrigation District (YRID)]. Disponible en: http://drryaqui.org.mx/antecedentes.html (última consulta el 18 de diciembre de 2018).

Evans, S. (2006). La angustia de La Angostura: consecuencias socioambientales por la construcción de presas en Sonora. [The angst of La Angostura: socio-environmental consequences for the construction of dams in Sonora]. Departamento de Historia. 


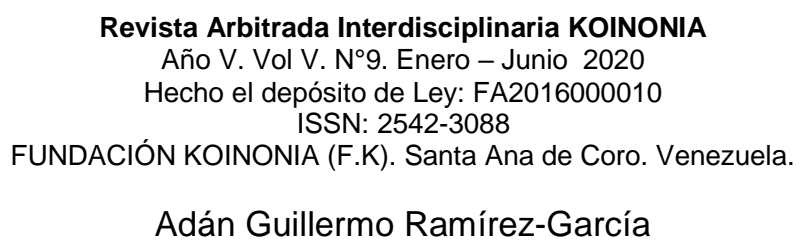

Brandon University. Disponible en: http://www.scielo.org.mx/pdf/sh/v8n16/1665-4420sh-8-16-46.pdf (última consulta el 31 de diciembre de 2018).

Fabio, A. et al. (2002). Manual Agropecuario. [Agricultural Manual]. Colombia. Ed. IBALPE.

FIRA. (2018). Sistema de costos agrícolas. Resumen de costos. Trigo. Agencia de Sonora. Ciclo Ol 18-19. Fideicomisos Instituidos en Relación con la Agricultura. [Agricultural cost system. Summary of costs. Wheat. Sonora Agency. Cycle Ol 18-19. Trusts Established in Relation to Agriculture]. Disponible en: https://n9.cl/rtoa. (última consulta el 2 de enero de 2019).

Gliessman S. (2002). Agroecología: procesos agroecológicos en agricultura sostenible. . [Agroecology: agroecological processes in sustainable agriculture]. Ed. Agruco. pp. 315.

INIFAP. (1999). El campo experimental Valle del Yaqui: su importancia en la producción agrícola del sur de Sonora. [Agroecology: agroecological processes in sustainable agriculture]. Instituto Nacional de Investigaciones Forestales, Agrícolas y Pecuarias. Cd. Obregón, Sonora. pp. 3-4, 18-21.

Márquez, B. S., et al. (2014). Trigo en Sonora y su contexto nacional e internacional. Centro de Estudios para el Desarrollo Rural Sustentable y la Soberanía Alimentaria. [Wheat in Sonora and its national and international context]. México, D.F. 218 p.

Masera, O., Astier, M., y López R. (2000). Sustentabilidad y Manejo de recursos naturales: El marco de la Evaluación MESMIS. [Sustainability and Natural Resource Management: The framework of the MESMIS Assessment]. México. D. F. Editorial Mundi-prensa.

Moreno-Vázquez, R. (1994a). Análisis de datos. [Data Analysis]. En: Moreno, R(Ed.). Sanidad vegetal en la horticultura protegida. Consejería de Agricultura y Pesca. Junta de Andalucía. Sevilla: 109-112.

Moreno-Vázquez, R. (1994b). Captura de datos. En: Moreno, R(Ed.). Sanidad vegetal en la horticultura protegida. [Data Capture. In: Moreno, R (Ed.). Plant health in protected horticulture] Consejería de Agricultura y Pesca. Junta de Andalucía. Sevilla: 93-106.

Okada, A. (2000). El impacto de la Revolución Mexicana: la Compañía Constructora Richardson en el valle del Yaqui (1905-1928). [The impact of the Mexican Revolution: the Richardson Construction Company in the Yaqui Valley (1905-1928).]. Historia Mexicana 197, pp. 91-143.

Padilla, C. E. (2014). Usuarios del agua en el Valle del Yaqui durante la gestión de la compañía Richardson, 1906-1919. Una caracterización sociocultural. [Water users in the Yaqui Valley during the management of the Richardson company, 1906-1919. A sociocultural characterization]. Secuencia, Revista de historia y ciencias sociales, núm. 89, pp. 27-54.

Picado, U. W. (2012). Conexiones de la Revolución Verde Estado y cambio tecnológico en la agricultura de Costa Rica durante el período 1940-1980. [Connections of the Green 
Revolution State and technological change in agriculture in Costa Rica during the period 1940-1980.]. Tesis. Universidade de Santiago de Compostela. Facultade de Xeografía e Historia. Departamento de Historia e Contemporánea de América. 428 p.

Sánchez, G. P. (2005). Problemática de la producción agrícola del Valle del Yaqui. [Problems of agricultural production in the Yaqui Valley]. Documento Interno, Centro Regional Universitario del Noroeste, Ciudad, Obregón, Sonora, México.

Salón, M., \& Isea Argüelles, J. (2019). El emprendimiento agroalimentario y políticas públicas en Venezuela. [Agri-food entrepreneurship and public policies in Venezuela] Revista Arbitrada Interdisciplinaria Koinonía, 4(8), 24-53. http://dx.doi.org/10.35381/r.k.v4i8.235

Sevilla, G. 2001. Una estrategia de sustentabilidad a partir de la agroecología. [A sustainability strategy based on agroecology]. Agroecología e Desenv. Rur. Suistent, Vol.2, N.1, p. 35-45.

Sonino, A., y Ruane, J. (2011). La innovación en agricultura como herramienta de la política de seguridad alimentaria: el caso de las biotecnologías agrícolas. Biotecnologías e innovación: el compromiso social de la ciencia. [Innovation in agriculture as a tool of food security policy: the case of agricultural biotechnologies. Biotechnologies and innovation: the social commitment of science].

Almanza, S. M. (2008). Social sector organizations of the Yaqui Valley. [Organizaciones del sector social del valle del Yaqui. Contratiempos de la política agrícola.]. Agricultural policy setbacks. Northern Border, Vol. 20, Núm. 40, 33 p.

Altieri, M. (1999). Agroecology: Scientific basis for Sustainable Agriculture. [Agroecología: Bases científicas para una agricultura sostenible]. Uruguay: Ed. Nordan-Comunidad.

Altieri, M. \& Nicholls, C. (2000). Agroecology: Theory and Practice for Sustainable Agriculture. [Agroecología: Bases científicas para una agricultura sostenible]. Basic Texts Series for Environmental Training. ONU-PNUMA.

Berber, D. S., Almaguer Vargas, G., Schwentesius Rindermann, R., \& Ayala Garay, A. V. (2014). Wheat in Sonora and its national and international context. [El trigo en Sonora y su contexto nacional e internacional]. Obregón, Sonora, México.

Bermejo, G. R. (2014). From sustainable development according to Brundtland to sustainability as biomimesis. [Del desarrollo sostenible según Brundtland a la sostenibilidad como biomimesis]. Basque Country University. Spain. 60 p.

Borbón, C. (2011). Diagnosis of the Agricultural and Fisheries Sector in Sonora State. [Diagnóstico del sector agrícola y pesquero en el estado de Sonora]. Center for Research in Food and Development, A.C.

Brunett P., L. (2004). Contribution to the evaluation of the sustainability: studies of two agroecosystems of milk production in Toluca Valley. [Contribución a la evaluación de la sostenibilidad: estudios de dos agroecosistemas de producción de leche en el valle 


\section{Revista Arbitrada Interdisciplinaria KOINONIA \\ Año V. Vol V. Nº. Enero - Junio 2020 \\ Hecho el depósito de Ley: FA2016000010 ISSN: 2542-3088 \\ FUNDACIÓN KOINONIA (F.K). Santa Ana de Coro. Venezuela. \\ Adán Guillermo Ramírez-García}

de Toluca]. Doctoral thesis. Veterinary Medicine and Zootechnics Faculty, National Autonomous University of Mexico. Mexico.

Cabello, T., \& Benítez E. (1994). Frankliniella occidentalis (Thysanoptera; Thripidae). In: Moreno, $\mathrm{R}(\mathrm{Ed}$.). General pest management in protected horticulture. [Frankliniella occidentalis (Thysanoptera; Thripidae Manejo general de plagas en horticultura protegida]. Ministry of Agriculture and Fisheries. Andalusian Board, Seville: 243-259.

Castillo, D. et al. (2012). Evaluation of the social, economic and productive sustainability of two small-scale milk production agroecosystems in Amecameca, Mexico. [Evaluación de la sostenibilidad social, económica y productiva de dos agroecosistemas de producción de leche a pequeña escala en Amecameca, México.]. UDO Agricultural Scientific Magazine. Vol. 12, No. 3, 2012, p. 690-704.

DRRY. (2018). Irrigation District of the Yaqui River history. [Distrito de riego de la historia del río Yaqui]. Available in: http://drryaqui.org.mx/antecedentes.html (accesed december 18, 2018).

Evans, S. (2006). The angst of La Angostura: socio-environmental consequences for the construction of dams in Sonora. History Department Brandon University. [La angustia de La Angostura: consecuencias socioambientales para la construcción de presas en Sonora.]. Available in: http://www.scielo.org.mx/pdf/sh/v8n16/1665-4420-sh-8-1646.pdf (accesed in december, 31, 2018).

Fabio, A. et al. (2002). Agricultural Manual. [Manual agrícola]. Colombia. Ed. IBALPE.

FIRA. (2018). Agricultural cost system. Summary of costs Wheat. Sonora Agency. AW cycle 18-19. Trusts Instituted in Relation to Agriculture. [Sistema de costos agrícolas. Resumen de costos Trigo. Agencia Sonora]. Available in: https://www.fira.gob.mx/InfEspDtoXML/TemasUsuario.jsp (accesed in january 2, 2019).

Gliessman S. (2002). Agroecology: agroecological processes in sustainable agriculture. [Agroecología: procesos agroecológicos en agricultura sostenible]. Ed. Agruco. p. 315.

INIFAP. (1999). The experimental field Yaqui Valley: its importance in agricultural production in southern Sonora. National Institute of Forestry, Agricultural and Livestock Research. [El campo experimental Valle del Yaqui: su importancia en la producción agrícola en el sur de Sonora. Instituto Nacional de Investigación Forestal, Agropecuaria]. Obregon City, Sonora, p 3-4, 18-21.

Márquez, B. S., et al. (2014). Wheat in Sonora and its national and international context. Center for Studies for Sustainable Rural Development and Food Sovereignty. [Trigo en Sonora y su contexto nacional e internacional. Centro de Estudios para el Desarrollo Rural Sostenible y la Soberanía Alimentaria]. Mexico, D.F. 218 p.

Masera, O., Astier, M., y López R. (2000). Sustainability and Natural Resource Management: The MESMIS Evaluation framework. [Sostenibilidad y gestión de 
Revista Arbitrada Interdisciplinaria KOINONIA

Año V. Vol V. Nº. Enero - Junio 2020

Hecho el depósito de Ley: FA2016000010 ISSN: 2542-3088

FUNDACIÓN KOINONIA (F.K). Santa Ana de Coro. Venezuela.

Adán Guillermo Ramírez-García

recursos naturales: el marco de evaluación MESMIS]. Mexico. D. F. Editorial Mundipress.

Moreno-Vázquez, R. (1994a). Analysis of data. [Análisis de datos]. In: Moreno, R (Ed.). General pest management in protected horticulture. Ministry of Agriculture and Fisheries. Andalusian Board, Seville: 109-112.

Moreno-Vázquez, R. (1994b). Data Capture. [Captura de datos] In: Moreno, R (Ed.). General pest management in protected horticulture. Ministry of Agriculture and Fisheries. Andalusian Board, Seville: 93-106.

Okada, A. (2000). The impact of the Mexican Revolution: the Richardson Construction Company in Yaqui Valley (1905-1928). [El impacto de la Revolución Mexicana: compañía de construcción Richardson en el Valle del Yaqui (1905-1928)] Mexican History 197, pp. 91-143.

Padilla, C. E. (2014). Water users in the Yaqui Valley during the direction of the Richardson Company, 1906-1919. A sociocultural characterization. [Usuarios de agua en el Valle del Yaqui durante la dirección de la Compañía Richardson, 1906-1919.Una caracterización sociocultural]. Sequence, Magazine of history and social sciences, no. 89 , pp. $27-54$.

Picado, U. W. (2012). Connections of the Revolution Green. State and technological change in the agriculture of Costa Rica during the period 1940-1980. . [Conexiones de la Revolución Verde. Estado y cambio tecnológico en la agricultura de Costa Rica durante el período 1940-1980]. Thesis. University of Santiago de Compostela. Geography and History Faculty. Department of History and Contemporary America, p. 428.

Sánchez, G. P. (2005). Agricultural production problems in Yaqui Valley. [Problemas de producción agrícola en el valle de Yaqui]. Internal Document, Northwest University Regional Center, City, Obregón, Sonora, Mexico.

Sevilla, G. (2001). A sustainability strategy based on agroecology. [Problemas de producción agrícola en el valle de Yaqui]. Agroecology and sustainable rural development, Vol. 2, No. 1, p. 35-45.

Sonino, A., y Ruane, J. (2011). Innovation in agriculture as a tool for food security policy: the case of agricultural biotechnologies. Biotechnologies and innovation: the social commitment of science. [La innovación en la agricultura como herramienta para la política de seguridad alimentaria: el caso de las biotecnologías agrícolas. Biotecnologías e innovación: el compromiso social de la ciencia.]. Available in: https://n9.cl/56zq. (accesed in december 12, 2018).

(C2020 por los autores. Este artículo es de acceso abierto y distribuido según los términos y condiciones de la licencia Creative Commons Atribución-NoComercial-Compartirlgual 4.0 Internacional (CC BY-NC-SA 4.0)

(https://creativecommons.org/licenses/by-nc-sa/4.0/). 